\title{
RELIEFF FEATURE SELECTION BASED ALZHEIMER DISEASE CLASSIFICATION USING HYBRID FEATURES AND SUPPORT VECTOR MACHINE IN MAGNETIC RESONANCE IMAGING
}

\author{
Halebedu Subbaraya Suresha \\ Department of ECE, Don Bosco Institute of Technology, \\ Kumbalgodu, Mysore Road, Bangalore, India \\ Dr. S.S. Parthasarathi \\ Professor, EEE Department, \\ PES College of Engineering, Karnataka, India
}

\begin{abstract}
Alzheimer disease is a form of dementia that results in memory-related problems in human beings. An accurate detection and classification of Alzheimer disease and its stages plays a crucial role in human health monitoring system. In this research paper, Alzheimer disease classification was assessed by Alzheimer's disease Neuro-Imaging Initiative (ADNI) dataset. After performing histogram equalization and skull removal of the collected brain images, segmentation was carried-out using Fuzzy C-Means (FCM) for segmenting the white matter, Cerebro-Spinal Fluid (CSF), and grey matter from the pre-processed brain images. Then, hybrid feature extraction (Histogram of Oriented Gradients (HOG), Local Binary Patterns (LBP), and Gray-Level CoOccurrence Matrix (GLCM)) was performed for extracting the feature values from the segmented brain images. After hybrid feature extraction, reliefF feature selection was used for selecting the optimal feature subsets or to reject the irrelevant feature vectors. Then, the selected optimal feature vectors were given as the input to a supervised classifier Support Vector Machine (SVM) to classify three Alzheimer classes of subjects; those are normal, Alzheimer disease and Mild Cognitive Impairment (MCI). The experimental outcome showed that the proposed methodology performed effectively by means of sensitivity, accuracy, specificity, and f-score. The proposed methodology enhanced the classification accuracy up to 2-20\% compared to the existing methodologies.
\end{abstract}

Keywords: Alzheimer Disease, Fuzzy C-Means, Gray-Level Co-Occurrence Matrix, Histogram of Oriented Gradients, Local Binary Patterns, Support Vector Machine. 
Cite this Article: Halebedu Subbaraya Suresha and Dr. S.S. Parthasarathi, Relieff Feature Selection Based Alzheimer Disease Classification using Hybrid Features and Support Vector Machine in Magnetic Resonance Imaging, International Journal of Computer Engineering and Technology, 10(1), 2019, pp. 124-137.

http://iaeme.com/Home/issue/IJCET?Volume=10\&Issue=1

\section{INTRODUCTION}

Alzheimer disease is a neurological disorder that damages the brain cells and leads to memory loss and other mental diseases. Currently, $5 \%$ of people in total population are suffering from Alzheimer disease [1-2]. The cause of Alzheimer disease ineffectively understood and still, no proper treatment exposed for stopping the progression of the disease. An early diagnosis of Alzheimer disease helps in determining its progression and improve the quality of life of Alzheimer disease patients. Still, it is a challenging process for accurate diagnosis of Alzheimer disease in the clinic [3-4]. Multimodality neuro-images like positron emission tomography and Magnetic Resonance Images (MRI) provides useful imaging information to understand the functional and anatomical neural changes related to Alzheimer disease [5]. In that, MRI is a non-invasive medical imaging modality, which is utilized for imaging the internal body structures. The MRI uses radio frequency pulses and magnetic field for producing detailed images of bones, soft tissues, organs, and other internal body structures [68]. Nowadays, MRI scans particularly used in brain imaging, where it delivers information about the morphology of the gray matter, white matter and CSF [9]. Therefore, it is necessary to develop new methodologies for supporting clinicians, and for the personalized detection of disease. Alzheimer disease is the common neuro-degenerative disease in older people. There is a considerable delay between the clinical diagnosis of Alzheimer disease and the start of Alzheimer disease pathology. In addition, it is very difficult to detect Alzheimer disease early and to support clinicians in the personalized diagnosis of the disease [10]. To overcome these issues, a computerized Alzheimer disease classification system is developed.

Earlier, many research studies carried out to improve the classification accuracy of Alzheimer disease, but still the conventional methods can't achieve a satisfactory result. In this experimental research, an effective methodology is proposed to improve the classification accuracy of Alzheimer disease. At first, the brain images were collected from the dataset (ADNI). The unwanted noises in the collected brain images were eliminated by employing histogram equalization. The respective pre-processed brain images were used for skull removal using Otsu thresholding approach. After skull removal, segmentation was carried out using FCM for segmenting the white matter, CSF, and grey matter. Then, hybrid feature extraction was applied to the segmented regions for extracting the feature values. Hybrid feature extraction was the procedure of obtaining feature subsets from the set of input data by the rejection of redundant and irrelevant features. After obtaining the feature information, the reliefF feature selection was utilized to select the optimal feature subsets. The output of reliefF feature selection specifies the features of segmented regions, which were essential for classification. These optimal feature values were given as the input for SVM to classify the three Alzheimer classes of subjects: normal, Alzheimer disease and MCI. At last, performance of the proposed methodology was compared with the existing methodologies by means of sensitivity, accuracy, specificity, and f-score. The contribution of the proposed work comprises of following points.

- Hybrid feature extraction based classification performed by combining descriptor level features and texture features.

- While performing both hybrid feature extraction and classification, it delivered improved results on multi-class and binary classification. 
- The proposed system classified three Alzheimer subjects using binary classification, which is an inexpensive process compared to multi-class classification.

This research paper is composed as follows. Section II surveys several recent papers on Alzheimer disease classification. In section III, an effective supervised methodology is proposed for Alzheimer disease classification. Section IV shows quantitative and comparative analysis of proposed and existing methodologies. The conclusion is made in section $\mathrm{V}$.

\section{LITERATURE REVIEW}

Recently, the researchers in Alzheimer disease classification developed numerous research methodologies. A brief evaluation of a few essential contributions to the existing literatures is presented in this sub-section.

T. Altaf et al., [11] presented an effective Alzheimer detection and classification algorithm. Initially, a bag of visual word methodology was utilized for improving the efficiency of texture features like LBP, scale invariant feature transform, GLCM and HOG. Here, the clinical data along with imaging data were highlighted by combining the texture features with clinical features for generating the hybrid feature vectors. The extracted features from the segmented region of MRI brain image represents the CSF, grey matter and white matter. This methodology was evaluated by using the dataset ADNI. The experimental section confirmed that this methodology outperformed the existing methodologies in terms of specificity, accuracy, and sensitivity. The major drawback of the developed methodology is that it is very difficult to identify the free space projection using this technique. In texturebased feature extraction, free space projection can able to deliver a better discriminant ability.

Z. Sun et al., [12] developed a new SVM based learning methodology for Alzheimer disease classification. In the developed SVM classification methodology, the spatial neighbour features in the anatomical regions have same weights. Then, a group of lasso penalty was introduced for inducing structure sparsity that helped physician for assessing the key regions involved in the disease. In this literature, an accelerated proximal gradient descent methodology was developed for solving the learning problem. This experimental research was carried out on an online database; ADNI. The extensive experiments were conducted and the effectiveness of the developed methodology was verified by means of specificity, accuracy, sensitivity, and area under a curve. The learning methodology utilized in this research was too sensitive to the parameters that highly affects performance of the classification.

I. Beheshti et al., [13] presented an effective approach for Alzheimer disease classification, which is the combination of novel feature selection and t-test based feature ranking. The developed system involved in five phases; voxel based morphometry approach used for comparing the local global differences of gray matter, the differences in gray matter volume selected as Volume of Interests (VOIs), 3) voxel clusters employed as VOIs, features ranked using t-test scores, ranked features classified using SVM, and data fusion methodology utilized for enhancing the classification performance. The experimental section confirmed that the developed system more effective than the existing systems in terms of specificity, accuracy, sensitivity and area under the curve. However, in a large dataset this methodology failed to achieve better classification result.

T. Tong et al., [14] presented a multi-modality classification system for exploiting complementarity in the multi-modal data. Initially, a pairwise similarity was used to determine the modality of features like CSF biomarker measures, categorical genetic information, regional MRI volumes, and voxel based signal intensities. Then, the similarities from multi-modality were combined with a non-linear graph fusion mechanism for generating a unified graph for classification. Experimental analysis and verification confirmed that this 
system effectively enhanced the performance of Alzheimer disease classification. In case, if the number of input samples becomes low, the multi-modality classification system gets affected and automatically the classification rate minimizes.

X. Tan et al., [15] developed an effective approach (Instance Transfer Ensemble Learning (ITL)) for Alzheimer disease classification. Initially, gravity transfer used for transferring the source domain data closer to the target data. Then, the best deviation between the target domain samples and the transferred source domain samples was searched by using ITL. At last, the target domain transferred samples and the optimal transferred domain samples were combined for classification. The developed methodology investigated by using the ADNI database. The experimental outcome confirmed that the developed methodology outperformed the existing methodologies in terms of standard deviation and mean. In this paper, Alzheimer disease classification done by manual adjustment, so it should be automated.

An effective supervised methodology is used in the current research work to overcome the above-mentioned drawbacks and to improve the classification of Alzheimer disease.

\section{PROPOSED MODEL}

In this research study, a new computer-aided design system is developed for enhancing the effectiveness and efficiency of Alzheimer disease classification. The proposed methodology is used for classifying three Alzheimer classes of subjects that has six major phases: image collection, pre-processing, segmentation, feature extraction, feature selection, and classification. The proposed methodology block diagram is presented in figure 1 . The brief description about the proposed methodology is detailed below.

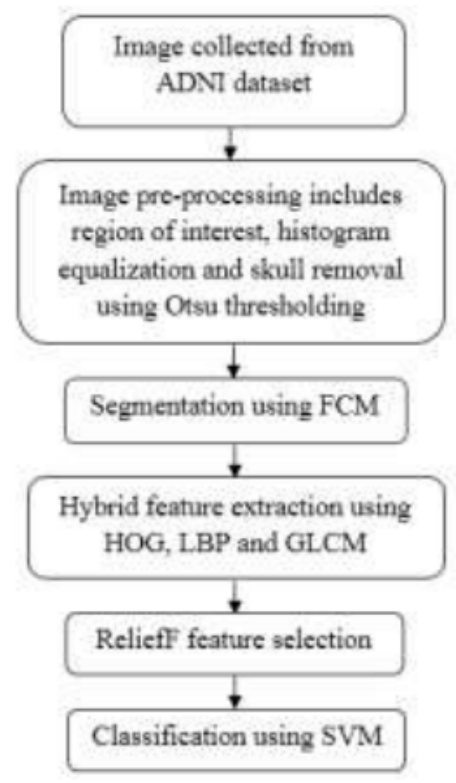

Figure 1 Working procedure of proposed methodology

\subsection{Image collection and pre-processing}

Initially, the brain images are collected from the ADNI dataset for Alzheimer disease classification. The ADNI database comprises of $3.0 \mathrm{~T}$ and $1.5 \mathrm{~T}$ t1 $\mathrm{w}$ MRI scans for MCI, normal controls, and AD at several time points. The general characteristics of ADNI dataset are detailed in the Table 1. 
Relieff Feature Selection Based Alzheimer Disease Classification using Hybrid Features and

Support Vector Machine in Magnetic Resonance Imaging

Table 1 Description about ADNI dataset

\begin{tabular}{|c|c|c|c|}
\hline Diagnosis & Age & Number & Gender (male /female) \\
\hline Alzheimer disease & Range [55-91] & 137 & $67 / 70$ \\
\hline Normal & Range [60-90] & 162 & $76 / 86$ \\
\hline MCI & Range [55-88] & 210 & $127 / 83$ \\
\hline
\end{tabular}

After collecting the images from ADNI dataset, a region of interest and preprocessing is carried out using histogram equalization. Pre-processing is used for enhancing the input image or reducing the noise in the collected brain image. There are numerous techniques used for pre-processing: smoothing, filtering, de-noising, etc. In this scenario, histogram equalization is utilized for pre-processing the brain images. It is a technique to adjust brain image intensities to enhance the image contrast. Let $f$ be a given brain image represented by matrix integer pixel intensities $m$ ranging from 0 to $L-1$. The histogram equalized brain image $g$ is defined in the Eq. (1).

$$
g_{i, j}=\text { floor }(L-1) \sum_{n=0}^{f(i, j)} p_{n}
$$

Where, $L$ is denoted as possible intensity value of 256 and $p$ is represented as normalized histogram of $f$ with a bin for each possible intensity, and floor () rounds down the nearest integers that are equivalent to transform the pixel intensities $k$, and it is expressed in the Eq. (2).

$$
T(k)=\text { floor }(L-1) \sum_{n=0}^{k} p_{n}
$$

In the transformation, the intensities $f$ and $g$ are considered as continuous random variables of $X=Y$ on $[0, L-1]$ with $Y$, which is defined in the Eq. (3).

$$
Y=T(X)=(L-1) \int_{0}^{x} p_{X}(x) d x
$$

Where, $p_{X}$ is represented as probability density function of $f, T$ is denoted as cumulative distributive function of $X$ multiplied by $(L-1)$, and $T$ indicates differentiable and invertible. It shows that $Y$ defined by $T(X)$ is uniformly distributed on $[0, L-1]$ namely $p_{Y}(y)=\frac{1}{L-1}$, which is expressed in the Eq. (4), (5), and (6).

$$
\begin{gathered}
\int_{0}^{y} p_{Y}(z) d z=\frac{1}{L-1}=\int_{0}^{T^{-1}(y)} p_{X}(w) d w \\
\frac{d}{d y}\left(\int_{0}^{y} p_{Y}(z) d z\right)=p_{Y}(y)=p_{X}\left(T^{-1}(y)\right) \frac{d}{d y}\left(T^{-1}(y)\right) \\
\frac{d T}{d x}{ }_{\mid x=T^{-1}(y)} \frac{d}{d y}\left(T^{-1}(y)\right)=(L-1) p_{X}\left(T^{-1}(y)\right) \frac{d}{d y}\left(T^{-1}(y)\right)=1
\end{gathered}
$$

Where, $p_{Y}(y)=\frac{1}{L-1}$

After histogram equalization, Otsu thresholding is performed for skull removal. The Otsu thresholding is a discriminate analysis, which is used for identifying the maximum separability of classes and to perform automatic histogram shape based image thresholding. The Otsu thresholding iterates and determines the pixel values that either fall in the background or foreground. The objective of this is to identify the threshold value, where the sum of background and foreground spreads at its minimum. After skull removal, segmentation done by FCM clustering for segmenting the white matter, CSF, and grey matter from the skull removed image. The sample input and pre-processed brain image is represented in the Fig. 2. 


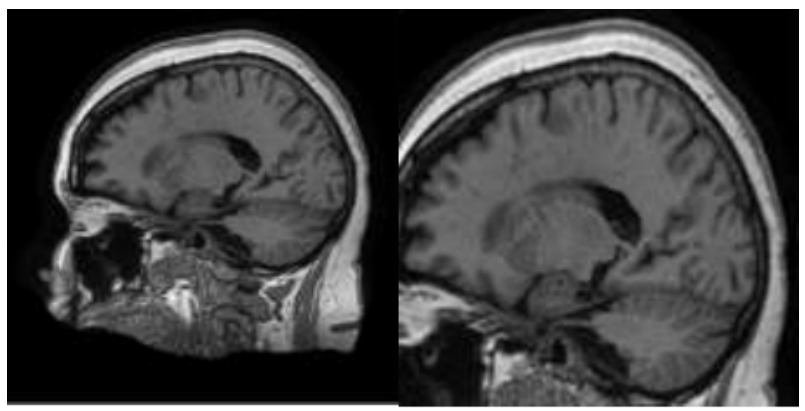

(a)

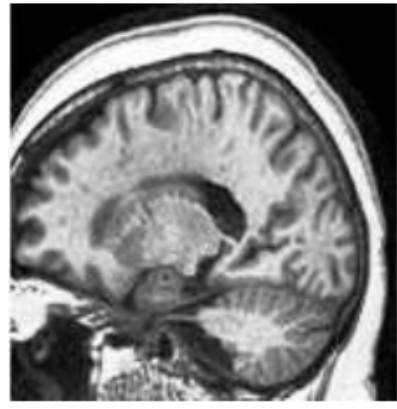

(c) (b)

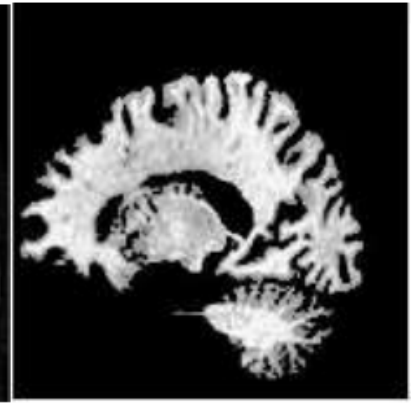

(d)

Figure 2 a) Input image, b) region of interest, c) pre-processed image, and d) skull removed image

\subsection{Segmentation using fuzzy c means}

In this research, FCM is used for segmenting the white matter, CSF, and grey matter from the brain images. In conventional segmentation methodologies, it is very difficult to segment the ill-defined portions that greatly decreases the segmentation accuracy. To address this issue, FCM is used in this research for localizing the object in complex template. Usually, FCM adopts fuzzy set theory for assigning a data object to more than one cluster. The FCM clustering considers each object as a member of every cluster with a variable degree of "membership". The similarity between the objects is defined by a distance measure, which plays an important role in obtaining correct clusters. In every iteration of FCM algorithm, the objective function $j$ is minimized, which is mathematically given in the Eq. (7).

$$
j=\sum_{i=1}^{N} \sum_{j=1}^{C} \delta_{i j}\left\|x_{i}-c_{j}\right\|^{2}
$$

Where, $C$ is represented as the clusters, $N$ is denoted as data points, $\delta_{i j}$ is stated as the degree of membership for the $i-t h$ data point $x_{i}$ in cluster $j$, and $c_{j}$ is represented as the centre vector of cluster $j$. The norm $\left\|x_{i}-c_{j}\right\|$ calculates the similarity of the data points $x_{i}$ to the centre vector $c_{j}$ of cluster $j$. For a given data $x_{i}$, the degree of membership $\delta_{i j}$ is calculated by using the Eq. (8).

$$
\delta_{i j}=\frac{1}{\sum_{k=1}^{c}\left(\frac{\left\|x_{i}-c_{j}\right\|}{\left\|x_{i}-c_{k}\right\|}\right)^{\frac{2}{m-1}}}
$$

Where, $m$ is denoted as fuzziness coefficient and the centre vector $c_{j}$ is determined by the Eq. (9).

$$
c_{j}=\frac{\sum_{i=1}^{N} \delta_{i j}^{m} \cdot x_{i}}{\sum_{i=1}^{N} \delta_{i j}^{m}}
$$


In the Eq. (8) and (9), the fuzziness coefficient $m$ calculates the tolerance of the clustering. The higher value of $m$ represents the larger overlap between the clusters. In addition, the higher fuzziness coefficient utilizes a larger number of data points, where the degree of membership is either one or zero. The degree of membership determines the number of iterations completed by the FCM clustering approach. Here, the accuracy $a$ is measured by using the degree of membership from one iteration $k$ to the next iteration $k+1$, which is calculated by the Eq. (10).

$$
a=\Delta_{i}^{N} \Delta_{i}^{C}\left|\delta_{i j}^{k+1}-\delta_{i j}^{k}\right|
$$

Where, $\Delta$ is represented as the largest vector value, $\delta_{i j}^{k+1}$ and $\delta_{i j}^{k}$ are denoted as the degree of membership of iterations $k+1$ and $k$. The segmented white matter, CSF, and grey matter are graphically denoted in the Fig. 3. After segmentation, hybrid feature extraction is carried out for extracting the feature vectors from the segmented regions.

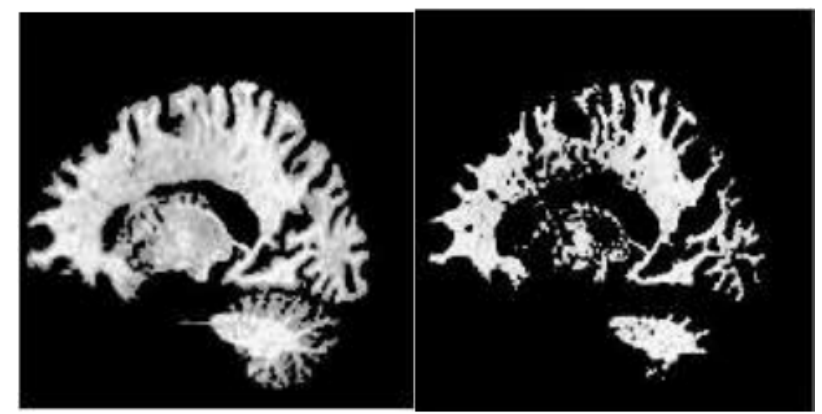

(a)

(b)

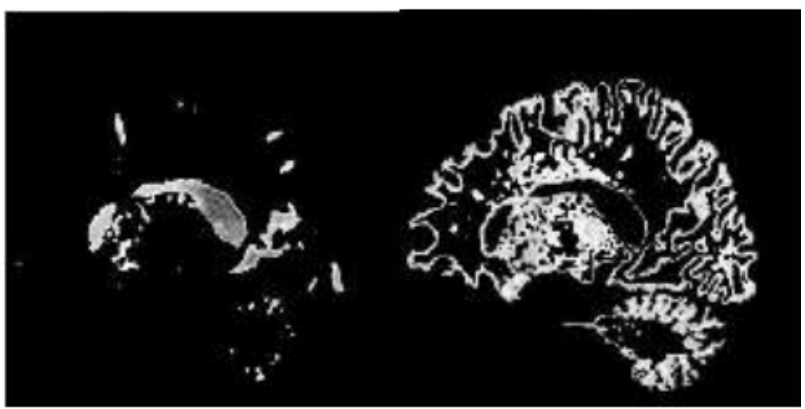

(c)

(d)

Figure 3 a) skull removed image, b) segmented white matter image, c) segmented CSF image, and d) segmented grey matter image

\subsection{Hybrid feature extraction}

Usually, feature extraction is defined as the action of mapping the brain image from image space to feature space and it transforms the large redundant data into a reduced data representation. It helps to decrease the complexity of the system. In this research study, feature extraction is performed by using HOG, LBP and GLCM features in order to extract the feature values from the segmented regions. The detailed description about the feature descriptors are given below.

\subsubsection{Histogram of oriented gradients}

Usually, HOG describes the distribution of spatial directions in each brain image region. It exploits the local object appearance, which is well characterized by the distribution of edge directions or local intensity gradients. 
The general idea of HOG is to divide the image into small spatial regions and for each region, it creates a one-dimensional gradient orientation histogram with gradient direction and magnitude. A key characteristic of HOG feature is capable of capturing the local appearance of objects to account the invariance in object transformations and illumination condition. The edge information about gradients is determined by applying the HOG feature vector. At first, a gradient operator $N$ is employed to calculate the gradient value. The gradient point of the brain image is denoted as $(x, y)$, which is mathematically expressed in the Eq. (11).

$$
G_{x}=N * I(x, y) \text { and } G_{y}=N^{T} * I(x, y)
$$

Image detection windows are categorized into various minor spatial regions, which is known as cells. Hence, the magnitude gradients of the pixels undergone with edge orientation. Finally, the magnitude of the gradients $(x, y)$ is denoted in the Eq. (12).

$$
G_{x}(x, y)=\sqrt{G_{x}(x, y)^{2}}+\sqrt{G_{y}(x, y)^{2}}
$$

Edge orientation of the point $(x, y)$ is specified in the Eq. (13).

$$
\theta(x, y)=\tan ^{-1} \frac{G_{y}(x, y)}{G_{x}(x, y)}
$$

Where, $G_{x}$ is mentioned as the horizontal direction of gradients and $G_{y}$ is represented as the vertical direction of gradients.

After the calculation of histogram values, a normalization procedure performed for superior invariance in illumination and noise. Normalization is an essential step in the HOG feature descriptor, it maintains discriminative characteristics and performs consistently even against parameters like background-foreground contrast and local illumination variations in the input brain image. Normalization is done by using "block" as a fundamental region of operation. Each block region comprises of a square array of four cells. Each new block is defined with a $50 \%$ overlap with the previous block. Normalization effectively maintains the cell-based local gradient information, which is invariant to local illumination conditions. In HOG, four different patterns of normalizations are available; those are L2-norm, L2-Hys, L1Sqrt, and L1-norm. Among these normalizations, L2-norm delivers better performance in Alzheimer detection and classification, which is mathematically given in the Eq. (14).

$$
L_{2-n o r m}: \mathrm{f}=\frac{x}{\sqrt{\|x\|_{2}^{2}+e^{2}}}
$$

Where, $e$ is denoted as small positive value, $f$ is represented as feature extracted value, $x$ is meant as non-normalized vector in histogram blocks and $\|x\|_{2}^{2}$ represents the 2-norm of HOG normalization.

\subsubsection{Local binary pattern}

LBP is a texture analysis descriptor that transforms an input brain image into labels based on the luminance value. In LBP, gray-scale invariance is an essential factor that depends on the texture and local patterns. In a brain image $f$, the pixel position is mentioned as $x$ and $y$ that is derived by using the central pixel value $x_{c}$ of $x$ as the threshold to signify the neighbourhood pixel $m$ value. The binary value of the pixel is weighted by using the power of two and then summed to create a decimal number to store in the location of central pixel $x_{c}$, which is mathematically given in the Eq. (15).

$$
\operatorname{LBP}(x, y)=\sum_{i=0}^{m-1} f\left(x_{i}-x_{c}\right)^{2 i}, f(x)=\left\{\begin{array}{l}
1, x \geq 0 \\
0, x \leq 0
\end{array}\right\}
$$


Where, $x_{i}$ stated as the gray level value of the central pixel of a local neighbourhood. The basic neighbourhood model of LBP is ( $p$-neighbourhood model) that gives $2^{p}$ output, which leads to a large number of possible patterns. If the texture analysis descriptor area is small, the LBP histogram is not attractive. The uniform model of LBP attains only when the jumping time maximizes. It is measured by using the Eq. (16).

$$
U(L B P(x, y))=\left|f\left(x_{c-1}-x_{i}\right)-f\left(x_{0}-x_{i}\right)\right|+\sum_{i=1}^{m-1}\left|f\left(x_{c}-x_{i}\right)-f\left(x_{c-1}-x_{i}\right)\right|
$$

Where, $u$ is indicated as maximum jumping time.

\subsubsection{Gray level co-occurrence matrix}

The GLCM descriptor is utilized to determine the frequency of pixel pairs, when the pixel intensity values are equal. In this research study, GLCM descriptor comprises of twenty-one features: autocorrelation, contrast, correlation, cluster prominence, cluster shade, dissimilarity, energy, entropy, homogeneity, maximum probability, sum of squares, variance, sum average, sum variance, sum entropy, difference variance, difference entropy, information measure of correlation, inverse difference, inverse difference normalized and inverse difference moment normalized. Among these twenty-three features along with HOG and LBP, the optimal feature values are selected by using reliefF feature selection. A brief description about the reliefF feature selection is given in the below section.

\subsection{ReliefF feature selection}

Feature selection is a high-level process that identifies the relevant subsets of data based on a particular criterion. In feature selection, the mutual information between the features is calculated for identifying the optimal features that help to decrease the computational effort. In this research, reliefF feature selection is used for selecting the optimal feature vectors to perform better classification. The reliefF algorithm is very robust while dealing with noisy and incomplete data.

Initially, the reliefF algorithm randomly selects an instance $r_{i}$ and then searches for $k$ nearest neighbor for the same class is named as nearest hit $H$ and for dissimilar classes is named as nearest miss $M$. Then, it updates the quality estimation $W[A]$ for all attributes $A$ that depends on the values of $r_{i}, M$, and $H$. If the instances $H$ and $r_{i}$ have similar values of the attribute $A$ then the attribute $A$ is separated into two instances with the similar class, which is desirable to decrease the quality estimation $W[A]$. Instead of this, if the instances $H$ and $r_{i}$ have dissimilar values of the attribute $A$ then the attribute $A$ is separated into instances with the dissimilar class, which is desirable to increase the quality estimation $W[A]$. The whole mechanism repeat for $m$ times, where $m$ is represented as a user defined parameter. In this research, the user-defined parameter is fixed as 20 . In reliefF algorithm, the quality estimation $W[A]$ is updated using the Eq. (17).

$$
W[A]=W[A]-\frac{\sum_{k=1}^{k} D_{H}(k)}{m \cdot k}+\sum_{c=1}^{c-1} p_{c} \frac{\sum_{k=1}^{k} D_{M}(k)}{m \cdot k}
$$

Where, $p_{c}$ is represented as prior class, and $D$ is denoted as distance between the selected instances $r_{i}$.

\subsection{Classification using support vector machine}

After feature selection, classification is carried out by using SVM that enables an efficient way of extracting the features and a set of rules to perform classification. SVM is a discriminative classification approach represented by a separate hyper-plane. 
The SVM classifier widely used in several applications like Bioinformatics, signal processing, computer vision fields, etc., due to its high performance in accuracy, and ability to process the high dimensional data. SVM does well in solving the two-class problem, which is associated with the theories of vapnik-chervonenkis and structure principles. The general formula for the linear discriminant function is denoted as $w \cdot x+b=0$. In order to distinguish the samples without noise, an optimum hyper plane is exploited between the two groups, which is mathematically given in the Eq. (18).

$$
p i[w \cdot x+b]-1 \geq 0, i=1,2, \ldots N
$$

Then, reduce $\|w\|^{2}$ in the Eq. (18), so the optimization issue is solved by the saddle point of a Lagrange function with Lagrange multipliers $\alpha_{i}$. The ideal discriminant function is denoted in the Eq. (19),

$$
f(x)=\operatorname{sign}\left\{\left(w^{*} x\right)+b^{*}\right\}=\operatorname{sign}\left\{\sum_{i=1}^{N} \alpha_{i}^{*} \cdot p i\left(x_{i}^{*}-x\right)+b^{*}\right\}
$$

Finally, replace the interior product $\left(x_{i}^{*}-x\right)$ by a linear kernel function $k\left(x, x^{\prime}\right)$ in the Eq. (19) for reducing the computational complexity in higher dimensional data. In this way, the linear separability of estimated samples improved and the discriminant function is re-written as given in the Eq. (20).

$$
f(x)=\operatorname{sign}\left\{\sum_{i=1}^{N} \alpha_{i}^{*} \cdot p i \cdot k\left(x, x_{i}\right)+b^{*}\right\}
$$

\section{EXPERIMENTAL RESULTS AND DISCUSSION}

In this paper, the proposed methodology experimented using MATLAB (version 2016) with $3.0 \mathrm{GHz}$ Intel i5 processor. For determining the efficiency of proposed methodology, the performance of proposed methodology was compared with the existing methodologies (Partial Least Squares (PLS) + Principal Component Analysis (PCA) + SVM [16], Circular Harmonic Functions (CHFs) descriptors + Posterior Cingulate Cortex (PCC) [17], and fusion of volume [18]) on a reputed dataset ADNI. The performance of the proposed methodology is determined in terms of accuracy, specificity, sensitivity, and f-score.

\subsection{Performance metric}

The performance metric is defined as the measurement of outcomes, which develops a reliable information about the effectiveness and efficiency of the proposed methodology. The relationship between the input and output values of the proposed methodology understood by using the performance metrics like specificity, sensitivity, and f-score. The general formula for evaluating the specificity, sensitivity, and f-score are given in the Eq. (21), (22), and (23).

$$
\begin{aligned}
& \text { Specificity }=\frac{T N}{T N+F P} \times 100 \\
& \text { Sensitivity }=\frac{T P}{T P+F N} \times 100 \\
& F-\text { score }=\frac{2 T P}{2 T P+F P+F N} \times 100
\end{aligned}
$$

Additionally, accuracy is one of the effective evaluation measures used for finding the effectiveness of the proposed methodology of Alzheimer disease classification. Accuracy is the most instinctive performance measure and it is simply a ratio of total observations to the correctly predicted observations. The general formula of accuracy is given in the Eq. (24).

$$
\text { Accuracy }=\frac{T P+T N}{T P+T N+F P+F N} \times 100
$$

Where, $F P$ is signified as false positive, $T N$ is indicated as true negative, $T P$ is specified as true positive, and $F N$ idenotes false negative. 


\subsection{Quantitative analysis}

In this sub-section, ADNI dataset is assessed for evaluating the performance of the proposed methodology. In this research study, Alzheimer disease classification implemented on a digital image-processing platform to classify three Alzheimer classes of subjects: normal, Alzheimer disease and MCI. Here, the performance measures (accuracy, sensitivity, f-score and specificity) determined using 10-fold cross validation that helps to assess the discriminative accuracy of the different multivariate analysis. The 10 -fold cross validation considers the randomly selected sets of Alzheimer disease, MCI and normal. The 10-fold cross validation iteratively selects the testing data and train the classifier with the remaining sets. At first, train the computer-aided design system with normal and Alzheimer disease images (group 1). Next, the proposed methodology was tested using MCI and Alzheimer disease images (group 2) and finally train the computer-aided design system with Alzheimer disease images and $\mathrm{MCI}$ (group 3).

Table 2 Performance evaluation without reliefF feature selection

\begin{tabular}{|c|c|c|c|c|}
\hline \multicolumn{5}{|c|}{ Without reliefF feature selection } \\
\hline Diagnosis & Accuracy (\%) & Sensitivity (\%) & Specificity (\%) & F-score (\%) \\
\hline Normal vs Alzheimer disease & 80 & 80 & 80 & 80 \\
\hline Normal vs MCI & 75 & 60 & 90 & 78.26 \\
\hline MCI vs Alzheimer disease & 70 & 60 & 80 & 72.72 \\
\hline
\end{tabular}

In table 2, the proposed methodology performance validated in terms of sensitivity, specificity, accuracy, and f-score without reliefF feature selection. Here, the performance evaluation validated for 150 random brain images with $80 \%$ of training and $20 \%$ of testing. The average sensitivity and specificity of the proposed methodology without reliefF feature selection is $66.67 \%$ and $83.34 \%$. Similarly, the average accuracy and f-score of the proposed methodology without reliefF feature selection is $75 \%$ and $77 \%$. The graphical representation of the proposed methodology without reliefF feature selection is denoted in Fig. 4.

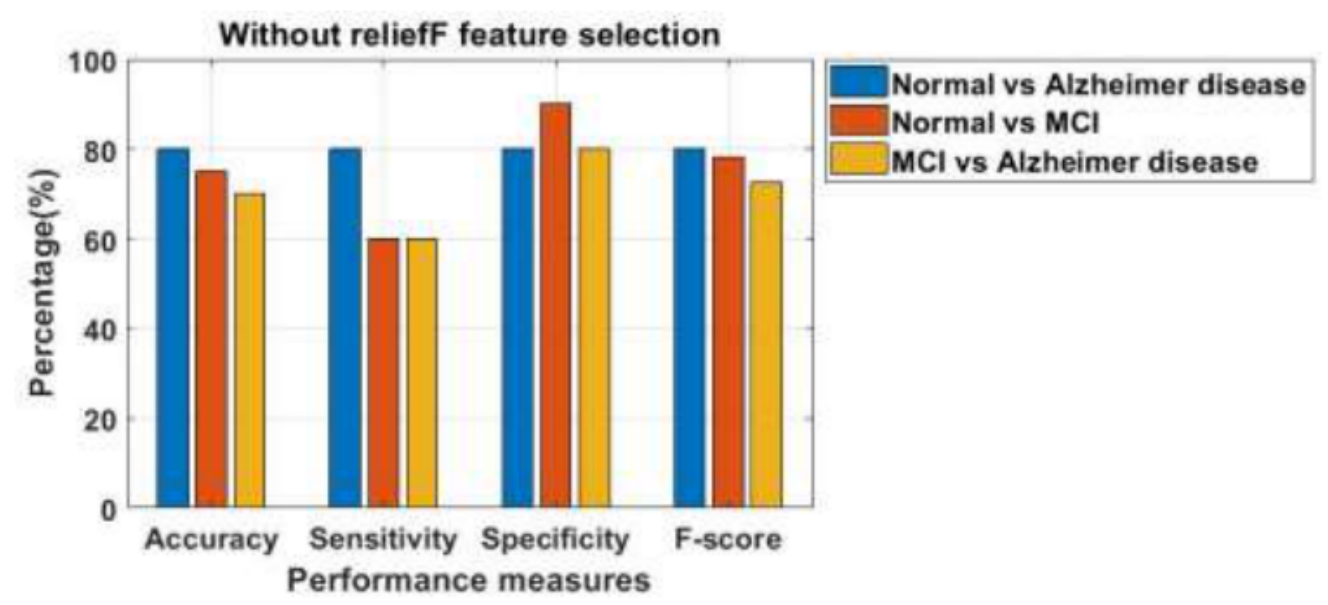

Figure 4 Graphical representation of proposed methodology without reliefF feature selection

In table 3, the proposed methodology performance evaluated in terms of sensitivity, specificity, accuracy, and f-score with reliefF feature selection. The average sensitivity and specificity of the proposed methodology with reliefF feature selection is $86.67 \%$ and $83.34 \%$. Correspondingly, the average accuracy and f-score of the proposed methodology with reliefF feature selection is $85 \%$ and $84.72 \%$. 
In with reliefF feature selection, the SVM classifier improves the accuracy in Alzheimer disease classification up to $10 \%$ compared to without reliefF feature selection. The graphical representation of proposed methodology with reliefF feature selection is denoted in Fig. 5.

Table 3 Performance evaluation with reliefF feature selection

\begin{tabular}{|c|c|c|c|c|}
\hline \multicolumn{5}{|c|}{ With reliefF feature selection } \\
\hline Diagnosis & Accuracy (\%) & Sensitivity (\%) & Specificity (\%) & F-score (\%) \\
\hline Normal vs Alzheimer disease & 90 & 90 & 90 & 90 \\
\hline Normal vs MCI & 85 & 100 & 70 & 82.35 \\
\hline MCI vs Alzheimer disease & 80 & 70 & 90 & 81.81 \\
\hline
\end{tabular}

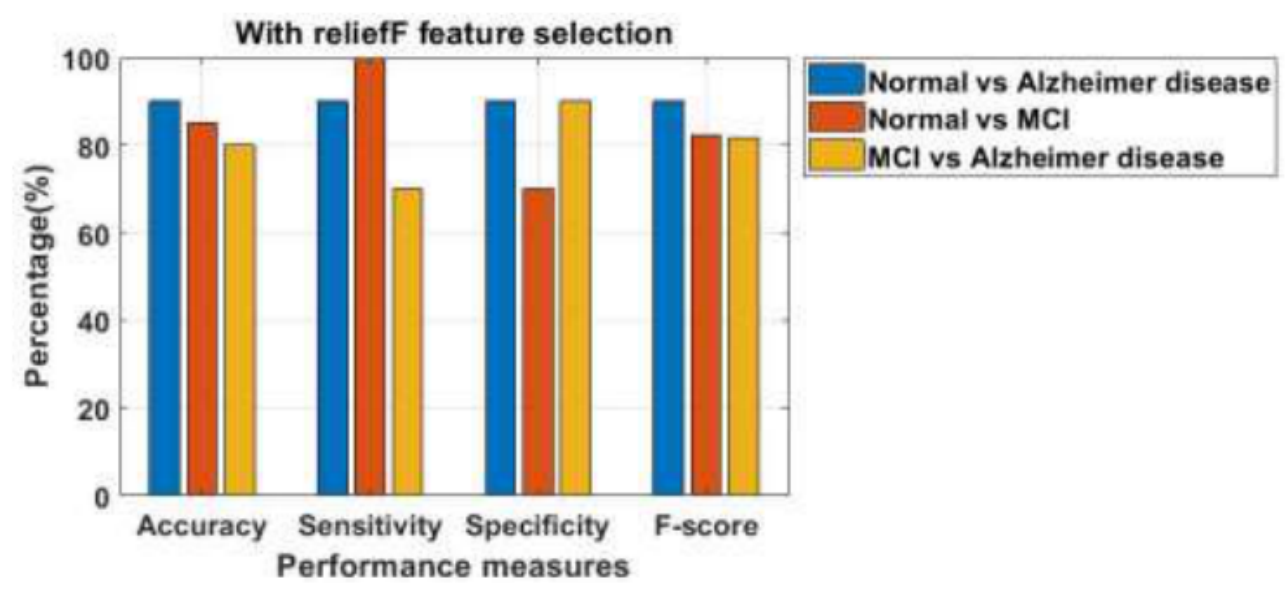

Figure 5 Graphical representation of proposed methodology with reliefF feature selection

\subsection{Comparative analysis}

The comparative study of the existing and proposed methodology is presented in the Tables 4 and 5. L. Khedher et al., [16] developed an effective Alzheimer disease classification system for classifying three Alzheimer classes of subjects: normal, Alzheimer disease and MCI. In this literature, PCA and PLS feature extraction approaches were used to extract the feature values from the collected brain images. After feature extraction, classification done by employing linear SVM classifier. This experiment was carried out on an online database (i.e. ADNI) to validate its result in terms of a classification accuracy, sensitivity and specificity. The developed methodology (PLS+ PCA + SVM) achieved maximum sensitivity of $85.11 \%$, specificity of $91.27 \%$ and accuracy of $88.49 \%$. Compared to this existing work, the proposed work achieved maximum sensitivity of $100 \%$, specificity of $90 \%$ and accuracy of $90 \%$ that was slightly higher than the existing work.

Table 4 Comparative analysis of proposed and existing methodology with maximum result

\begin{tabular}{|c|c|c|c|c|}
\hline Methodology & Database & Sensitivity (\%) & Specificity (\%) & Accuracy (\%) \\
\hline PLS+ PCA + SVM [16] & ADNI & 85.11 & 91.27 & 88.49 \\
\hline Propose methodology & ADNI & 100 & 90 & 90 \\
\hline
\end{tabular}

Additionally, O.B. Ahmed et al., [17] utilized pattern recognition and visual indexing framework for classifying three Alzheimer classes of subjects: normal, Alzheimer disease and MCI. In this research paper, CHFs was used for extracting the local features from PCC and hippocampus in every slice in all three-brain projection. Then, PCA and SVM approaches were used for feature dimensionality reduction and classification, respectively. 
This experiment was conducted on a subset of Bordeaux-3City and ADNI datasets. Here, this system almost achieved average $75.64 \%$ of accuracy.

In addition, X. Yang et al., [18] investigated the associations of CSF markers, shapes, and volumes of lateral ventricles and hippocampus with $\mathrm{AD}$ and $\mathrm{MCI}$ at the baseline. Then, apply baseline markers for predicting MCI conversion in a two-year time using ADNI dataset. This methodology averagely achieved $58 \%$ of sensitivity, $75 \%$ of specificity and $65 \%$ of accuracy. Compared to these existing methodologies, the proposed work averagely achieved $85 \%$ of classification accuracy, $83.34 \%$ of specificity, and $86.67 \%$ of sensitivity that was higher than the existing works. In this study, the hybrid features along with reliefF feature selection determines the non-linear and linear properties of brain image and preserves the quantitative relationships between the higher and lower level features. The evaluation metrics confirmed that the proposed methodology performed significantly in Alzheimer disease classification compared to the previous approaches.

Table 5 Comparative analysis of proposed and existing methodology with average result

\begin{tabular}{|c|c|c|c|c|}
\hline Methodology & Database & Sensitivity (\%) & Specificity (\%) & Accuracy (\%) \\
\hline CHFs descriptors + PCC [17] & ADNI & 72.24 & 68.94 & 75.64 \\
\hline Fusion of volume [18] & ADNI & 58 & 75 & 65 \\
\hline Proposed methodology & ADNI & 86.67 & 83.34 & 85 \\
\hline
\end{tabular}

\section{CONCLUSION}

In this research paper, an effective computer-aided diagnosis system is developed to assist the early detection and classification of Alzheimer disease. The proposed methodology is developed by combining three feature extraction methods (HOG, LBP, and GLCM) along with reliefF feature selection in order to enhance the classification of Alzheimer disease. The reliefF feature selection is used to select the optimal feature subsets or rejects the irrelevant feature vectors. This optimal feature information is given as the input for SVM classifier for classifying the three Alzheimer classes of subjects: normal, Alzheimer disease and MCI. The developed methodology helps the doctors/clinicians in diagnosing the Alzheimer disease effectively in terms of accuracy. Compared to other existing methods in Alzheimer disease classification, the proposed methodology delivered an effective performance by means of accuracy and shows $2-20 \%$ improvement in classification accuracy. In future work, a new unsupervised classification methodology can be implemented with high-level features for further improvement of the classification of Alzheimer disease.

\section{REFERENCES}

[1] T. Ye, C. Zu, B. Jie, D. Shen, D. Zhang, and Alzheimer's disease Neuroimaging Initiative, "Discriminative multi-task feature selection for multi-modality classification of Alzheimer's disease", Brain imaging and behavior, vol.10, no.3, pp.739-749, 2016.

[2] D. Baskar, V.S. Jayanthi, and A. N. Jayanthi, "An efficient classification approach for detection of Alzheimer's disease from biomedical imaging modalities" Multimedia Tools and Applications, pp.1-33, 2018.

[3] B. Magnin, L. Mesrob, S. Kinkingnéhun, M. Pélégrini-Issac, O. Colliot, M. Sarazin, B. Dubois, S. Lehéricy, and H. Benali, "Support vector machine-based classification of Alzheimer's disease from whole-brain anatomical MRI", Neuroradiology, vol.51, no.2, pp.73-83, 2009. 
[4] J. Escudero, E. Ifeachor, J.P. Zajicek, C. Green, J. Shearer, S. Pearson, and Alzheimer's Disease Neuroimaging Initiative, "Machine learning-based method for personalized and cost-effective detection of Alzheimer's disease", IEEE transactions on biomedical engineering, vol.60, no.1, pp.164-168, 2013.

[5] F. Liu, L. Zhou, C. Shen, and J. Yin, "Multiple kernel learning in the primal for multimodal Alzheimer's disease classification", IEEE J. Biomedical and Health Informatics, vol.18, no.3, pp.984-990, 2014.

[6] M. Liu, D. Cheng, K. Wang, Y. Wang, and Alzheimer's Disease Neuroimaging Initiative, "Multi-Modality Cascaded Convolutional Neural Networks for Alzheimer's Disease Diagnosis", Neuroinformatics, pp.1-14, 2018.

[7] O.B. Ahmed, J. Benois-Pineau, M. Allard, C.B. Amar, G. Catheline, and Alzheimer's Disease Neuroimaging Initiative, "Classification of Alzheimer's disease subjects from MRI using hippocampal visual features", Multimedia Tools and Applications, vol.74, no.4, pp.1249-1266, 2015.

[8] J. Liu, M. Li, W. Lan, F.X. Wu, Y. Pan, and J. Wang, "Classification of Alzheimer's Disease Using Whole Brain Hierarchical Network", IEEE/ACM transactions on computational biology and bioinformatics, vol.15, no.2, pp.624-632, 2018.

[9] Q. Zhou, M. Goryawala, M. Cabrerizo, J. Wang, W. Barker, D.A. Loewenstein, R. Duara, and M. Adjouadi, "An optimal decisional space for the classification of Alzheimer's disease and mild cognitive impairment", IEEE Trans. Biomed. Engineering, vol.61, no.8, pp.2245-2253, 2014.

[10] X. Liu, D. Tosun, M.W. Weiner, N. Schuff, and Alzheimer's disease Neuroimaging Initiative, "Locally linear embedding (LLE) for MRI based Alzheimer's disease classification", Neuroimage, vol.83, pp.148-157, 2013.

[11] T. Altaf, S.M. Anwar, N. Gul, M.N. Majeed, and M. Majid, "Multi-class Alzheimer's disease classification using image and clinical features", Biomedical Signal Processing and Control, vol.43, pp.64-74, 2018.

[12] Z. Sun, Y. Qiao, B.P. Lelieveldt, M. Staring, and Alzheimer's disease NeuroImaging Initiative, "Integrating spatial-anatomical regularization and structure sparsity into SVM: Improving interpretation of Alzheimer's disease classification", NeuroImage, 2018.

[13] I. Beheshti, H. Demirel, and Alzheimer's disease Neuroimaging Initiative, "Featureranking-based Alzheimer's disease classification from structural MRI", Magnetic resonance imaging, vol.34, no.3, pp.252-263, 2016.

[14] T. Tong, K. Gray, Q. Gao, L. Chen, D. Rueckert, and Alzheimer's disease Neuroimaging Initiative, "Multi-modal classification of Alzheimer's disease using nonlinear graph fusion", Pattern recognition, vol.63, pp.171-181, 2017.

[15] X. Tan, Y. Liu, Y. Li, P. Wang, X. Zeng, F. Yan, and X. Li, "Localized instance fusion of MRI data of Alzheimer's disease for classification based on instance transfer ensemble learning", Biomedical engineering online, vol.17, no.1, pp.49, 2018.

[16] L. Khedher, J. Ramírez, J.M. Górriz, A. Brahim, F. Segovia, and Alzheimer's Disease Neuroimaging Initiative, "Early diagnosis of Alzheimer's disease based on partial least squares, principal component analysis and support vector machine using segmented MRI images", Neurocomputing, vol.151, pp.139-150, 2015.

[17] O.B. Ahmed, M. Mizotin, J. Benois-Pineau, M. Allard, G. Catheline, C.B. Amar, and Alzheimer's Disease Neuroimaging Initiative, "Alzheimer's disease diagnosis on structural MR images using circular harmonic functions descriptors on hippocampus and posterior cingulate cortex", Computerized Medical Imaging and Graphics, vol.44, pp.1325, 2015.

[18] X. Yang, M.Z. Tan, and A. Qiu, "CSF and brain structural imaging markers of the Alzheimer's pathological cascade", PloS one, vol.7, no.12, pp.e47406, 2012. 